\title{
PLATELETS PREDICT PATIENT OUTCOME IN ISCHEMIC STROKE: THE SICFAIL (STROKE INDUCED CARDIAC FAILURE IN MICE AND MEN) COHORT STUDY
}

Mert Seyhan 1,2, Daniel Mackenrodt 1,2, Viktoria Rücker 1, Silke Wiedmann 1, Michael Schuhmann 2, Bernhard Nieswandt 3,4, Tobias Geisler ${ }^{5}$, Dominik Rath 5, Peter Kraft ${ }^{2,6}$, Christoph Kleinschnitz ${ }^{7}$, Peter Heuschmann 1,8

Background: Platelets are key players in the pathophysiology of ischemic stroke (IS). Their surface receptors mediate pathways of thrombosis, inflammation and repair. ${ }^{1,2}$ Measuring expression levels of certain receptors by flow cytometry can predict patient outcome in ischemic heart disease (IHD) ${ }^{3,4}$, but their prognostic role in stroke remains unclear.

Methods: Data collection was embedded in the ongoing prospective SICFAIL cohort study assessing the natural course of cardiac function after IS. In consecutive patients, blood was drawn and analyzed for expression of platelet markers by flow cytometry following standard procedure5: CXCR4, CXCR7, HMGB1, CD84, CD62p ( \pm ADP stimulation), CD40, CD41, GPV, GPVI, CD61 and CD42b. Expression was defined as mean fluorescence intensity (MFI) of events in the platelet gate (see Fig 1) in the PE channel (see Fig 2). In an exploratory approach logistic regression analysis was performed to assess the distinct associations between marker-MFI and the occurrence of poor outcome (NIHSS $\geq 5$ on day 3), adjusted for age and baseline NIHSS.

Results: 101 patients were included from 06/2016 until 01/2017 (see Tab 1).

Age, baseline NIHSS in combination with three distinct marker-MFIs were statistically significant associated with poor outcome (see Tab 2, Fig 3).

Tab 2: Logistic regression of poor outcome with age, baseline NIHSS and marker-MFI

\begin{tabular}{l|c|c|c} 
& OR & 95\%-Cl & p \\
\hline $\begin{array}{l}\text { Age [y] } \\
\begin{array}{l}\text { Baseline- } \\
\text { NIHSS }\end{array}\end{array}$ & 1.07 & 1.01 to 1.13 & 0.02 \\
\hline CXCR7-MFI & 3.52 & 1.12 to 1.50 & 0.01 \\
\hline HMGB1-MFI & 2.07 & 1.05 to 4.09 & 0.04 \\
\hline CD84-MFI & 1.27 & 1.04 to 1.57 & 0.02 \\
\hline
\end{tabular}

Fig 3: ORs (95\%-Cl) of marker-MFls in logistic regression of poor outcome (with age / baseline NIHSS)

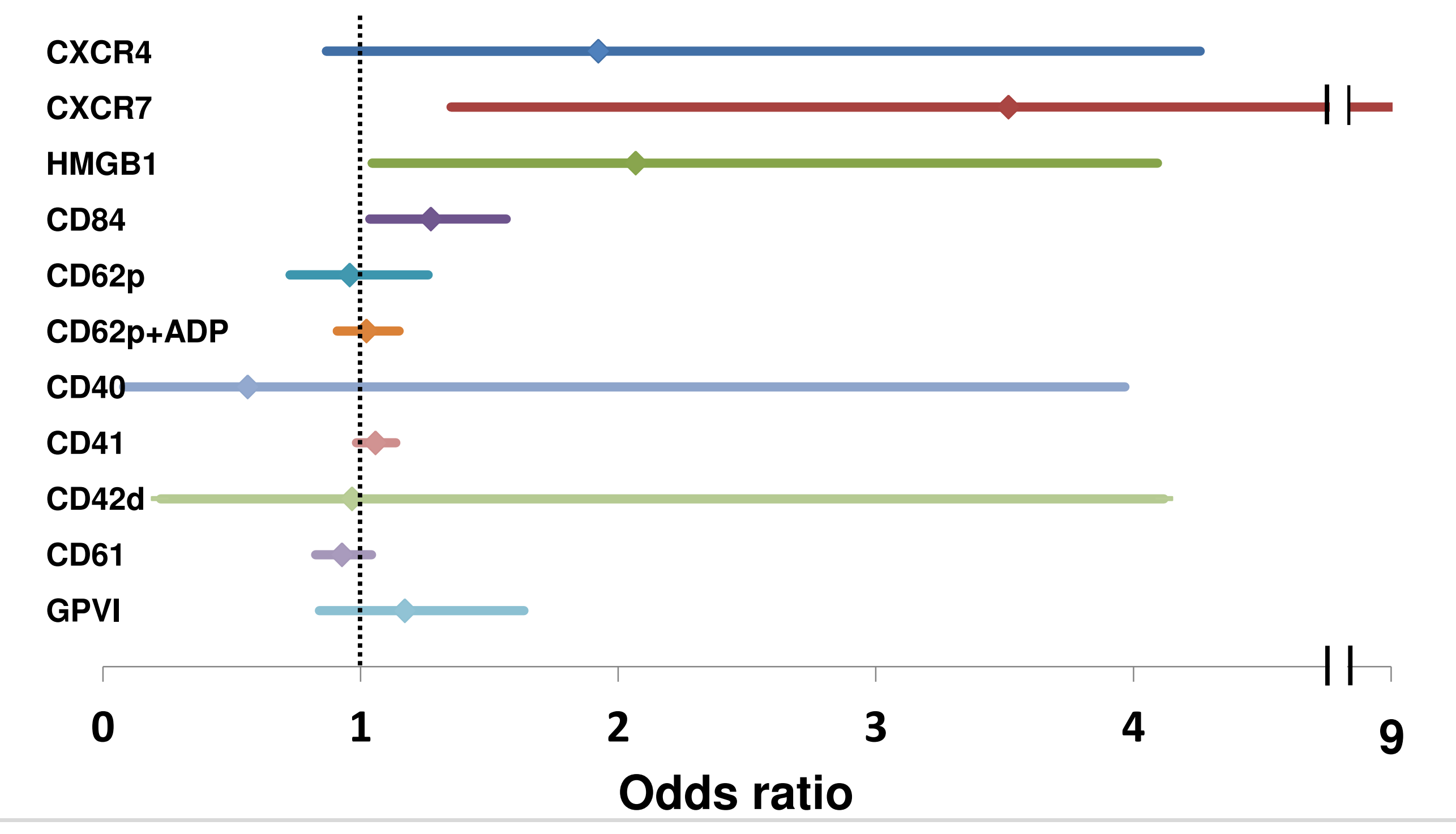

Limitations: The small sample size and the large marker panel lead to a high chance of false positive findings. The approach is based on the assumptions, that MFI equals expression and expression levels equal the functional level. Further there is a wide disparity in latency from stroke onset to platelet analysis. Lastly single markers provide only very weak individual prediction.

Conclusion: High MFI of CXCR7, HMGB1 and CD84 were linked to early unfavorable outcome. These results partly vary to published IHD data, where e.g. high CXCR7 appeared to be protective for early outcome. Underlying reasons might be organ-specific, methodological or chance, therefore replicating the data of this pilot study is required.

\section{References}

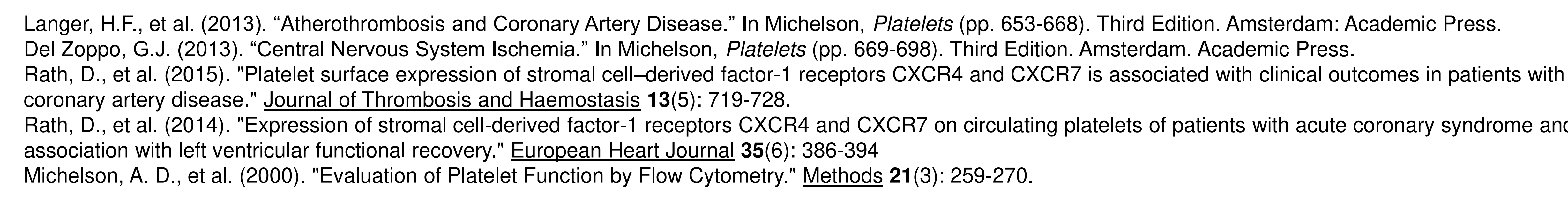

Fig 1: Gating strategy of platelets

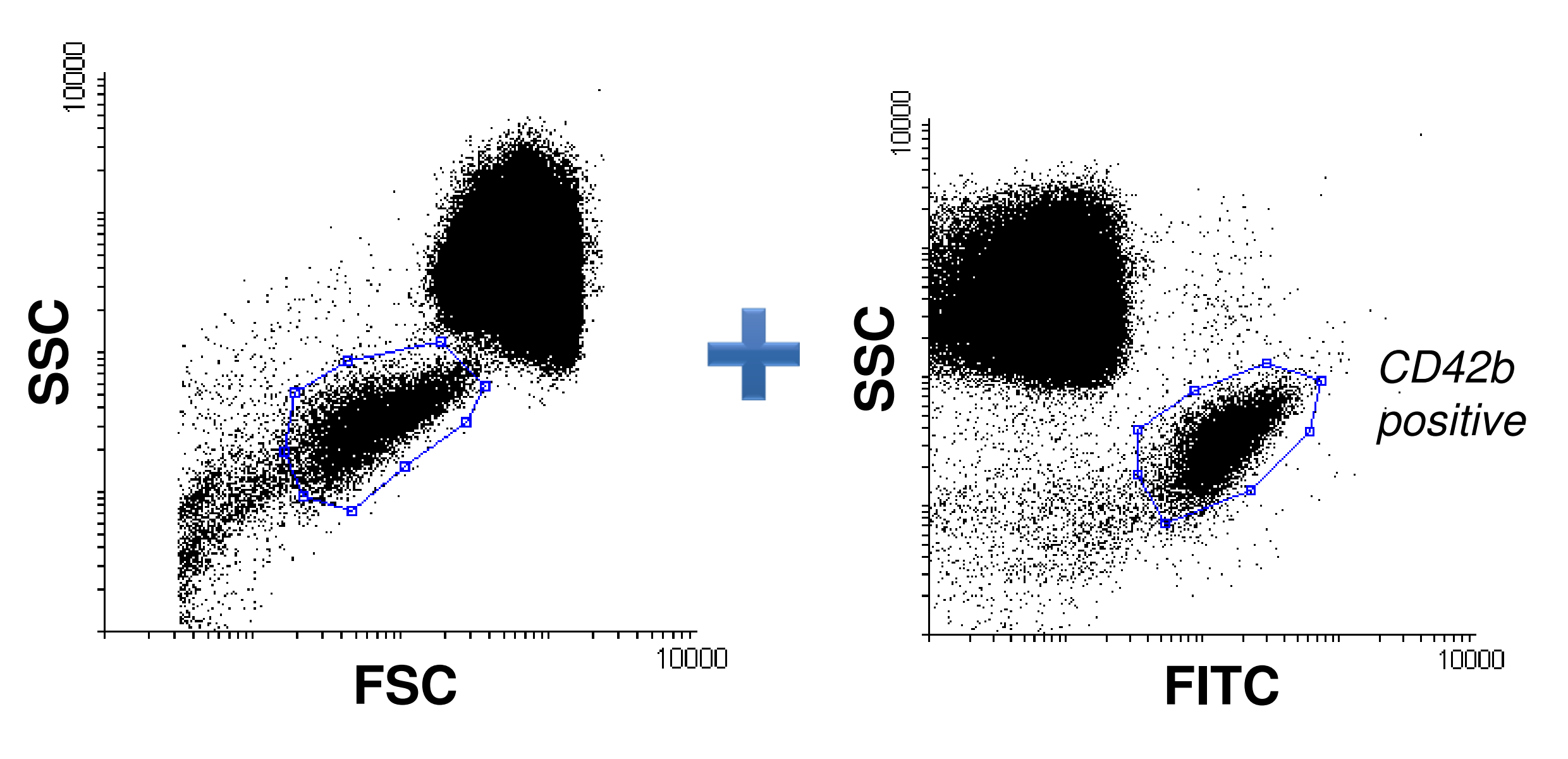

Fig 2: Example measurement CD62p \pm ADP

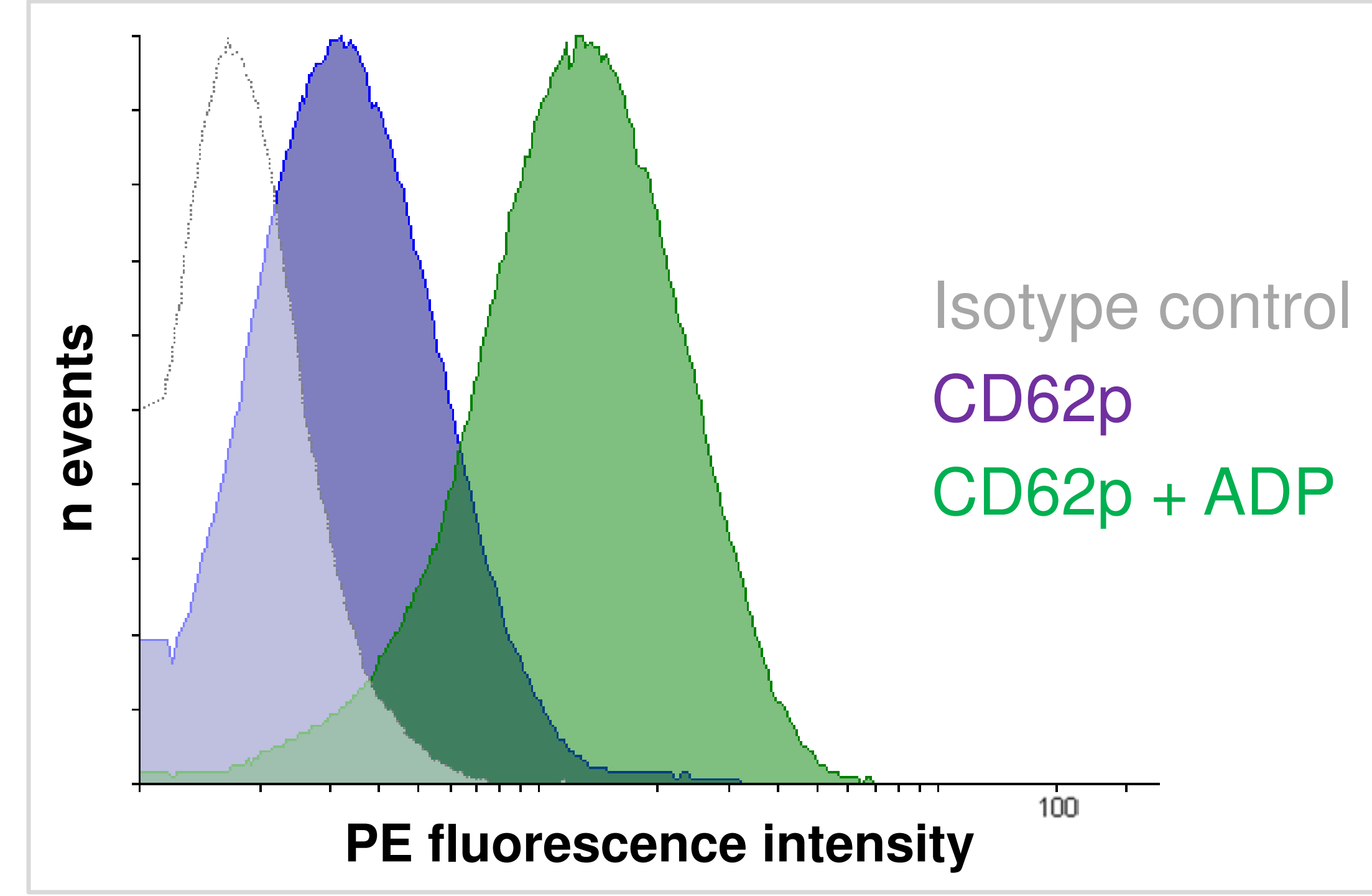

Tab 1: Patient characteristics $(n=101)$

\begin{tabular}{|l|c|c|}
\hline & Mean & SD \\
\hline Age [y] & 66 & 14 \\
\hline NIHSS day 1 & 5 & 4 \\
\hline NIHSS day 3 & 3 & 4 \\
\hline Latency stroke onset to & 76 & 35 \\
\hline blood draw [h] & & \\
\hline & $n$ & $\%$ \\
\hline Male sex & 66 & 65 \\
\hline Poor outcome & 19 & 19 \\
\hline Thrombolysis & 28 & 28 \\
\hline ASS & 77 & 76 \\
\hline Clopidogrel & 8 & 8 \\
\hline & Medical history & \\
\hline Stroke / TIA & 32 & 32 \\
\hline Myocardial infarction & 10 & 10 \\
\hline Atrial fibrillation & 16 & 16 \\
\hline Surgery last 30 days & 6 & 6 \\
\hline Active malignoma & 3 & 3 \\
\hline Chronic inflammation & 17 & 17 \\
\hline Smoking & 28 & 28 \\
\hline Diabetes mellitus II & 25 & 25 \\
\hline Hypertension & 85 & 84 \\
\hline
\end{tabular}

